Keio J. Med. 30: 11-15, 1981

\title{
SUSCEPTIBILITIES OF THE NORMAL BACTERIAL FLORA TO BROAD SPECTRUM ANTIBIOTICS UNDER ANAEROBIC CONDITION
}

\author{
TOSHIHIKO ARAI and YOKO KOMATSU \\ Department of Microbiology, School of Medicine, Keio University, \\ Tokyo, Japan
}

(Received for publication December 10, 1980)

\begin{abstract}
We measured the susceptibilities of the normal intestinal bacterial flora as well as opportunistic pathogens to the major broad spectrum antibiotics under anerobic conditions as was in intestine. The results showed that opportunistic pathogens were always more resistant to the commonly used antibiotics at that period than the commensal bacterial flora suggesting that abnormal increases of opportunistic pathogens caused by the uses of broad spectrum antibiotics could trigger the opportunistic infections in the immune deficient patients. Thus, the susceptibilities of the commensal bacteria to all antibiotics should be seriously considered for the adequate chemotherapy.
\end{abstract}

\section{INTRODUCTION}

Incidences of opportunistic infections have been increasing in these recent years in accordance with the increased usages of broad spectrum antibiotics. ${ }^{1}$ The opportunistic infections are thought to be due to the immune deficiency of the patients which have been mostly caused secondarily by anticancer, immunosuppressive or anti-inflammatory drugs. But the abnormal increases of opportunistic pathogens on dermal or mucosal surfaces could also play an important role for the infections of these weak virulent bacteria. ${ }^{2}$ Since the abnormal increases of these bacteria could be caused as the result of the death of the antibiotic sensitive commensal normal bacterial flora and the compensatory growth of these relatively antibiotic resistant bacteria, we had to be careful not to cause these changes of bacterial flora for the chemotherapy on immune deficient patients. For this purpose therefore, we should know the antibiotic susceptibilities of all normal flora. 
There are enough informations of antibacterial activities of every antibiotics against all pathogens including opportunistic pathogens, but no informations are available for antibacterial activities of the antibiotics against the major bacterial flora including obligate anaerobes in intestine.

In this paper, we described the minimal inhibitory concentrations (MICs) of the major broad spectrum antibiotics against various bacteria which could grow in human intestine ${ }^{3}$ and discussed the role of these antibiotics for the opportunistic infections.

\section{MATERIALS AND METHODS}

The strains employed for this study are listed in the Table 1. Obligate anaerobes were kindly supplied by Dr. T. Mitsuoka of the Institute of Physical and Chemical Research, Wako, Saitoma. Two standard strains for antibiotic susceptibility test given by National Institute of Health, Japan were used. Others were our stock strains originally isolated from clinical materials.

Aerobic and facultative anerobic bacteria were precultured in Trypticate soy broth (BBL) at $37^{\circ} \mathrm{C}$ for an overnight and diluted with the same fresh broth. Obligate anaerobic bacteria were precultured in the GAM soft agar tube (Nissui) at $37^{\circ} \mathrm{C}$ for an overnight and diluted with trypticase soy broth. $0.025 \mathrm{ml}$ of these diluted cultures (about $10^{4}$ cells) were spotted on GAM agar plates containing series of two fold diluted antibiotics and incubated at $37^{\circ} \mathrm{C}$ for 24 hours under anaerobic condition in GAS PAK jar (BBL). Streptomycin, kanamycin, tetracycline, chloramphenicol, cephalexin and ampicillin were used for MIC measurements. The concentrations which allowed no growth were taken as MICs.

\section{RESULTS AND DISCUSSION}

MICs of streptomycin, kanamycin, tetracycline, chloramphenicol, cephalexin and ampicillin against various intestinal flora under anaerobic condition are shown in the Table 1. Since normal intestine is anaerobic and containing high concentration of sulfhydroxyl compounds, ${ }^{4}$ MICs of the broad spectrum antibiotics were measured under anaerobic condition using the medium which contained the high amount of sulfhydroxyl compounds. Pseudomonas, Flavobacterrium, Acinetobacter and Alcaligenes are obligate aerobic bacteria and grow not well under anaerobic condition. But MICs against these bacteria were also measured in GAS PAK condition on GAM agar, because these condition is thought to be closer to the natural condition in intestine.

Intestine, especially caecum and colon are the biggest organs which allow 
Table 1

Minimal inhibitory concentrations of the major broad spectrum antibiotics against various bacteria

\begin{tabular}{|c|c|c|c|c|c|c|}
\hline Bacteria Antibiotic & SM & KM & TC & $\mathrm{CP}$ & $\mathrm{ABPC}$ & $\mathrm{CEX}$ \\
\hline Staphylococcus aureus NIHJ JC1 & 50 & 50 & 0.4 & 6.25 & 0.1 & 3.1 \\
\hline Staphylococcus epidermidis & $>1600$ & $<1600$ & 3.2 & 100 & 25 & 25 \\
\hline Streptococcus pyogenes & 6.25 & 12.5 & 0.8 & 1.6 & 0.1 & 0.1 \\
\hline Streptococcus faecalis & 200 & 200 & 100 & 50 & 0.4 & 200 \\
\hline Escherichia coli NIHJ JC2 & 50 & 50 & 0.8 & 1.6 & 0.2 & 6.25 \\
\hline Salmonella typhimurium & 50 & 100 & 3.1 & 6.25 & 1.6 & 25 \\
\hline Klebsiella pneumoniae & 25 & 50 & 6.25 & 6.25 & 800 & 6.25 \\
\hline Klebsiella oxytoca & 50 & $>1600$ & 1.6 & 6.25 & $>1600$ & 100 \\
\hline Enterobacter cloacae & $>1600$ & $>1600$ & 12.5 & $>200$ & $>1600$ & $>400$ \\
\hline Serratia marcescens & $>1600$ & $>1600$ & 25 & 200 & $>1600$ & $>400$ \\
\hline A eromonas hydrophila & 100 & 100 & 0.8 & 3.1 & 200 & 400 \\
\hline Pseudomonas aeruginosa & 100 & 200 & 25 & 50 & 800 & 0.2 \\
\hline Alcaligenes faecalis & 25 & 6.25 & 12.5 & 0.8 & 0.8 & 100 \\
\hline Flavobacterium meningo & 800 & 400 & 25 & 25 & 200 & 200 \\
\hline Acinetobacter anitratus & 400 & 6.25 & 0.8 & 25 & 25 & 200 \\
\hline Clostridium perfringens $\mathrm{T}-1$ & 1600 & 800 & 0.2 & 6.25 & 0.4 & 3.1 \\
\hline Clostridium difficile $\mathrm{V}-6$ & 400 & 800 & 25 & 3.1 & 0.8 & 200 \\
\hline Peptococcus asaccharolyticus $\mathrm{V}-23$ & 200 & 200 & 0.4 & 3. & 0.2 & 0.4 \\
\hline Veillonella parvula V-75 & 25 & 50 & 0.8 & 0.4 & $<0.1$ & $<0.1$ \\
\hline Propionibacterium acnes IX -62 & 6.25 & 400 & 0.8 & 0.4 & 0.1 & 0.4 \\
\hline Bifidobact rium longum IV-25 & 25 & 1600 & 3.1 & 1.6 & 0.8 & 25 \\
\hline entis IV -40 & 50 & 400 & 0.8 & 0 . & 0.1 & 0.1 \\
\hline ium bifidum IV-2 & $>1600$ & 400 & 1.6 & 1.6 & 0.1 & 12.5 \\
\hline Eubacterium lentum V-21 & 25 & 25 & 0.4 & 3.1 & 0.8 & 0.2 \\
\hline Eubacterium limosum V-60 & 100 & $>1600$ & 0.4 & 0.8 & 0.1 & 3.1 \\
\hline Eubacterium aerofaciens $\mathrm{V}-61$ & $>1600$ & 50 & 0.2 & 1.6 & 1.6 & 0.8 \\
\hline les fragilis $\mathrm{T}-2$ & 1600 & $>1600$ & 6.25 & 1.6 & 6.25 & 25 \\
\hline fragilis VI-24 & 1600 & $>1600$ & 12.5 & 3.1 & 25 & 200 \\
\hline Bacteroides thetaiotaomicron VI-25 & 800 & $>1600$ & 3.1 & 3.1 & 25 & 100 \\
\hline Bacteroides distasonis VI-28 & 400 & $>1600$ & 0.8 & 6.25 & 6.25 & 100 \\
\hline
\end{tabular}

Streptomycin, kanamycin, tetracycline, chloramphenicol, ampicillin and cephalexin are abbreviated as SM, KM, TC, CP, ABPC and CEX, respectively.

the highest growth of various bacterial flora. Antibiotics administrated reach to these organs not only directly by oral administration but by bilial circulation after intra-muscular and -venous injection and are consumed there by many drug sensitive bacteria resulting the death of these bacteria. But, because of the high amounts of bacteria flora, antibiotic concentrations decrease rapidly and, relatively resistant bacteria could survive and rather increase compensatory on the places formerly occupied by the killed bacteria. Thus, relative susceptibilities for the broad spectrum antibiotics should be important for the changes of 
bacterial flora from commensal bacteria to weak virulent opportunistic pathogens which produce tissue damaging proteolytic enzymes and cause local inflammation only when they are in high concentrations. In other words, the drugs which are less bacteriocidal to the major commensal intestinal flora, such as Bacteroides fragilis, Bifidobacterium bifidum and some Eubacterium spp. than opportunistic pathogens are thought to be safer drugs.

Comparing MICs of the major broad spectrum antibiotics against various intestinal flora from this point of view, it was suggested that continuous administration of streptomycin and kanamycin had almost no chance to allow selective growth of opportunistic pathogens, that administration of tetracycline could select Serratia, that administration of chloramphenicol could select Serratia, Pseudomonas, Flavobacterium and Acinetobacter, that administration of cephalexin could select Serratia, Klebsiclla oxytoca and Aeromonas, and that administration of ampicillin could select Serratia, K. oxytoca, Pseudomonas, Aeromonas and Acinetobacter. Because of the high incidences of $\mathrm{R}$ plasmids in bacterial flora, this order of relative resistances to these drugs could be modified in actual individuals. But these results are good correlation with the clinical cases of opportunistic pathogens, although incidences are also dependent on the frequency of colonization of each bacterial species in human intestine. ${ }^{1}$

An another antibiotic induced disease, antibiotic associated pseudomembranous colitis is known to be due to the death and the resulted release of toxin of Clostridium difficile in intestine caused by antibiotics..$^{5}$ This is also an another cause of intestinal inflammation. Since inflammation give chance to bacterial flora to invade into lymph and blood, this could be an another cause of opportunistic infections. This is also dependent on the relative antibiotic susceptibility of $C$. difficile.

MICs of various drugs against $C$. difficile suggested that streptomycin and kanamycin had no chance to cause the antibiotic associated colitis, but that tetracycline, chloramphenicol and ampicillin could cause this colitis. And this was also confirmed by the fact that the reported major causative antibiotics of this colitis were ampicillin and clindamycin. ${ }^{6}$

Because of the development of chemotherapy, mortalities as well as incidences of the serious bacterial infections were greatly reduced or disappeared for this decade. But, in contrast, incidences of opportunistic infections followed by the chemotherapy of the primary infections have been increasing and in most cases, further extensive chemotherapy for the first opportunistic infections resulted to the secondary and untreatable opportunistic infections.

As suggested by our works, the candidates of opportunistic pathogens are the bacteria that are more resistant to the used antibiotics than the commensal 
bacterial flora, and that produce proteases or other agents which cause tissue damages and local inflammations. Therefore, we should know which bacteria produce such agents and the relative antibiotic susceptibilities of these bacteria and the commensal bacterial flora. We already reported the list of protease producing bacteria which caused tissue damages when they grew up to a high concentrations. ${ }^{2}$ And in this paper, we also described the relative susceptibilities of these bacteria and the commensal bacteria for the major broad spectrum antibiotics.

There had been no information of the susceptibilities of the commensal bacterial flora for most of the antibiotics. But these are the most important information for chemotherapy, and the antibiotics which are more bacteriocidal for the commensal bacteria than opportunistic pathogens should not be used for the patients who are immune deficient, or are receiving anticancer, immunosuppressive or anti-inflammatory drugs.

Opportunistic infections are iatrogenic diseases in the sence that they are induced by chemotherapy. And it is possible to reduce the incidences by careful choices of drugs. We hope this paper will contribute for better understandings of the role of antibiotics for these infections and trigger the new developments of better drugs.

\section{REFERENCES}

1. Kobayashi, A., Tomioka, S. and Uchida, H.: Bacteria isolated from bloods. The Saishinigaku. 34: 1398-1405, 1979 (in Japanese)

2. Arai, T., Komatsu, S. and Komatsu, Y.: Extracellular protease production of various bacteria and the role of proteases on the pathogenicity of opportunistic pathogens. Keio J. Med. 30: 1-9, 1981 in press.

3. Mitsuoka, T.: A Color Atlas of Anaerobic Bacteria. Sobunsha, Tokyo, 1980 (in Japanese)

4. Arai, T.: Susceptibilities of bacteria against chemotherapeutics under different conditions. Keio J. Med. 26: 31-42, 1977

5. Bartlett, J. G.: Antimicrobial agent-associated pseudomembraneous colitis in patients. In: Microbiology-1979. edited by Schlessinger, D. American Society for Microbiology, Washington D.C. 1979, 264-266

6. Fekety, R.: Prevention and treatment of antibiotic-associated colitis. In: Microbiology-1979. edited by Schlessinger, D. American Society for Microbiology, Washington D.C. $1979,276-279$ 